\title{
异彩纷呈, 创意无限一一学音乐课堂打击乐器应用多样化刍议
}

\author{
文沫 黎嘉酒 \\ 江西师范大学音乐学院
}

DOI:10.32629/er.v3i4.2685

\begin{abstract}
[摘 要] 随着国家经济的快速发展和人民生活水平的不断提高,人们对丰富内心世界和精神世界的追求也越来越高。音乐艺术作为提高人们生 活品质的重要手段之一, 其价值也日益彰显出来。小学音乐课堂中器乐教学的引入, 不仅丰富了课堂教学的内容, 更为学生表现力、创造力和合 作交流能力的开发和基本音乐素养的养成提供了有效的载体。在课堂乐器的选择上,造价低廉、技巧性相对较低、使用范围广泛、益于激发学 生兴趣的打击乐器尤其受到音乐教师的青睐。笔者在对打击乐进课堂的价值、发展现状分析基础上,结合实践经验, 提出课堂打击乐器应用多样 化的几种可能性,旨在为丰富小学音乐课堂教学,进一步完善音乐表现及创造领域教学开拓新的思路。
\end{abstract}

[关键词] 打击乐; 多样化; 伴奏; 小学音乐课堂

作为表现领域的重要内容, 在小学音乐课堂中引入器乐教学, 不仅有 效丰富了课堂教学的内容, 更为学生表现力、创造力和合作交流能力的开 发和基本音乐素养的养成提供了平台。在课堂乐器的选择上, 造价低廉、 技巧性相对较低, 使用范围广泛、益于激发学生兴趣的打击乐器尤其受到 音乐教师的青睐, 小型打击乐器更是课堂教学中应用最广泛, 最简便易学 且最受学生欢迎的乐器。打击乐器不仅能够作为节奏训练的工具, 还可以 作为学习音乐各种要素、概念的教学媒介和工具, 在打击乐器的独奏、重 奏活动中培养学生的音乐感, 领悟、表现和理解音乐的能力及与人交流的 能力。

笔者在对大量小学音乐课堂教学案例研究中发现, 打击乐器进小学音 乐课堂的应用在世界各地都已十分普遍, 但我国多数音乐教师在打击乐器 的选用上较为单调, 仅使用铃鼓、三角铁、木鱼、沙锤等小型打击乐器, 应用方面, 也多限于为乐曲编配伴奏的传统形式。在小学音乐课堂教学手 段已极为丰富的今天, 打击乐进课堂的发展水平可以说与音乐教学发展大 环境不相匹配。故笔者在对打击乐进课堂的价值、发展现状分析基础上, 结合实践经验, 提出课堂打击乐器应用多样化的几种可能性, 旨在为丰富 小学音乐课堂教学, 进一步完善音乐欣赏、表现及创造领域教学开拓新的 思路。

\section{1 打击乐器进小学音乐课堂的价值}

打击乐器也叫 “敲击乐器”, 是指敲打乐器本体而发出声音的乐器。 其中有些是有固定音高的打击乐器, 如民族打击乐的云锣、编钟; 以及西 洋打击乐的马林巴、钟琴、木琴等; 其它还有一些无固定音高的打击乐器, 如民乐的拍板、梆子、板鼓、腰鼓、铃鼓; 西乐的小军鼓等。从广义上说， 通过击打身体不同部位发声的 “身体打击乐” 也成为使用频率很高的一类 音乐课堂打击乐器。

常见打击乐器具有简单、易上手、节奏性强的特点, 不需要经过长时 间的练习就能操作使用。因此在对比其它乐器有年龄限制和需要长期练习 才能使用的属性来看, 在小学音乐课堂中适度引进打击乐器, 在开发学生 综合素养方面有一定优势和实用价值。

1. 1 在兴趣引导下积累音乐鉴赏知识与能力

在审美性、人文性与实践性兼备的音乐课堂上, 除感受与欣赏教学之 外, 表现、创造等领域的教学内容同样值得关注, 在小学音乐课堂教学中适 度引入打击乐器, 既能有效提升教学内容的综合性, 更能引导学生在实践 中感受音乐的美感。

小学阶段, 尤其是小学低年级的学生, 尚不具备掌握复杂乐器的能力, 而常规打击乐器的使用正是小朋友们力所能及的。在许多的课堂实例中,
我们也发现学生擅长运用打击乐器完成各种编创活动。孩子们在聆听音乐 的同时, 能随音乐敲击各类打击乐器进行伴奏, 在充满乐趣的音乐课堂表 现活动中感受不一样的音乐美, 从多角度欣赏和体验音乐, 提升音乐鉴赏 能力。

\section{2在创新实践中树立正确的节奏感知}

为旋律编创打击乐伴奏是小学音乐课堂中的常见教学手段。在此类 环节中, 小朋友不仅要关注乐曲音高的变化, 更需要深入感知蕴含在旋 律进行中的节奏变化和节拍的强弱规律。为旋律选编伴奏的音乐实践, 也加深了小朋友对节奏的把控和变换能力, 帮助其逐步树立正确的节奏 感知。

在打击乐的伴奏织体中, 节奏型可以适当的进行变换。每换一种节奏 型, 就可以让一首乐曲在不改变曲谱的情况下, 形成另一种风格。这也造就 了打击乐伴奏的多样性, 为创新性思维能力的开发提供了可能。许多教学 案例中, 教师要求小朋友依据自己对音乐的理解和感受, 编创出自己想要 的音乐风格。一首乐曲, 可以进行一到两条不同风格的节奏编创; 可以使 用同一种节奏风格来配合不同的乐曲; 也可将多条节奏型组合, 改变强拍 的位置, 体验不同的节奏感觉, 在不断变换的乐曲和节奏编创中, 有效提升 学生的创新思维能力。

1.3 在集体活动中养成良好的社交能力

打击乐器自身特性决定它能在不影响主体音响效果的情况下, 为乐曲 润色。打击乐器不仅能为乐器和乐曲伴奏, 也能结合自身特色进行重奏, 相互之间进行声部间的配合, 在各类常规打击乐器演奏时的合作交流, 需 要同学之间的默契配合。强拍弱拍的乐器选择, 噪音与乐音的有效结合, 旋律中打击乐的进入与退出, 对指挥做出及时正确的反映等, 都需要集体 通力合作才能完成, 在这样经常性的集体活动中, 学生的社会交往能力和 与人合作的能力都将得到有效锻炼和提升。

\section{2 打击乐器进小学音乐课堂的现状与实施建议 \\ 2.1 发展现状}

虽然打击乐器种类繁多, 许多都具备操作简单使用便捷的特点, 但在 许多音乐教师的观念中, 打击乐器多为噪音乐器, 在表现教学领域中的器 乐教学, 他们更偏重于选择类似坚笛这类旋律乐器。另一方面, 在现今的小 学音乐课堂上, 使用较多的仍是一些较为简单的单一发声方式的打击乐器, 如: 木鱼、沙锤、铃鼓、三角铁等; 在教学设计中针对打击乐的应用环节 也较为单调, 多集中在给乐曲编配伴奏或简单的节奏教学上, 并没有真正 意义上的挖掘打击乐器的多样性及多用性, 亦无法体现打击乐器在创造力 开发和音乐综合素养培养方面的重要价值。最后, 在人们印象中, 打击乐器 
多为伴奏乐器, 无法进行主旋律演奏。对打击乐器种类的认识和了解不足 导致在教学设施的选择上, 大部分学校只购买极为简单的打击乐器作为伴 奏用途, 而音色优美, 有着固定音高的打击乐器鲜有学校进行购置, 这就造 成小学音乐课堂打击乐器种类单一, 使用频率甚低, 大大降低了打击乐器 能够为音乐课堂带来的作用。

\section{2 实施建议}

除我国传统打击乐器之外, 有许多在国外教学体系中应用普遍, 已经 有成套使用经验且适宜在小学课堂推广使用的打击乐器, 如: 非洲鼓、卡 宏箱、小木琴等。这些乐器价格适中, 使用也比较简单方便, 学生稍加学习, 便能轻松掌握。

相较于传统打击乐器或单调或音响效果粗糙或外观朴素或专业性 过强的特点, 这些适用于小学课堂的打击乐器种类多样, 更好看的外形、 更优美动听、更简单易上手、适用性更广等特点更能吸引学生。小学生 对新事物的的接受程度多来源于兴趣, 而此类打击乐器恰恰能够满足小 朋友对于新事物的新鲜感, 同时也为音乐教师的器乐教学提供更广阔的 平台。

这些种类繁多的打击乐器, 能为乐曲提供更加丰富的伴奏形式, 同时 由于乐器特征, 他们相互间还能进行独奏和重奏的组合, 为创新性思维能 力的开发提供了更多的可能。以 “涵养美感、和谐身心” 为主旨的小学音 乐课堂中, 形式种类多样化的打击乐器, 在普及音乐知识、提高音乐素养、 提升音乐学习兴趣等方面的效果显而易见, 丰富打击乐器种类的选择, 优 化打击乐器的使用, 能够极大程度增加音乐课堂的活力。

学校在配置音乐教具时, 可以更多关注种类繁多, 形式多样的打击 乐器。另外, 为适应不同年龄使用者, 这类打击乐器多提供针对小学生的 缩小版或定制版, 价格相较于成人版乐器便宜很多, 并不会增加过多的 采购经费。

\section{3 特色打击乐器进小学音乐课堂的实践研究}

在课堂打击乐器种类丰富的前提下, 音乐课堂教学形式也将极大丰 富。笔者将以非洲鼓、小木琴和身体打击乐为例, 探讨特色打击乐器使用 的可能性和常规方法。

3. 1 非洲鼓

尽管非洲鼓在我国小学音乐课堂使用并不普遍, 但作为最为常见的打 击乐器之一, 对小学音乐课堂来说, 有着非常广泛的适用性。其体积小巧, 音色多样、表现力丰富, 极具感染力。无论是乐曲伴奏、歌唱伴奏、还是 重奏都有着良好的演奏效果。

在课堂教学环节中, 非洲鼓可以作为节奏认知和节奏感培养的最佳手 段之一。小朋友可以在教师的指导下, 通过击打鼓的不同位置, 更清晰地了 解和感受强弱和音色, 更可以在集体合作的多线条节奏型中聆听体验丰富 多彩的节奏变化。

在乐曲欣赏环节中, 若以非洲鼓代替木鱼、三角铁之类的传统课堂打 击乐器, 用于伴奏时, 它将不再仅仅是强调正拍和反拍的工具, 其音响更为 丰富, 伴奏音型更加复杂, 能够通过更加符合乐曲的伴奏形式, 融入音乐; 而 即便脱离旋律用于独奏时, 非洲鼓的节奏击打也不会单调无味, 极易激发 学生演奏的兴趣。

在课堂活动环节中, 学生可以进行非洲鼓的即兴演奏, 也可以进行分 组合作演奏。在这里, 列举两种非洲鼓合作演奏方式为课堂教学提供参考。

\subsection{1圆圈模式}

小朋友以 4-6人一组, 围成一个圆, 同时演奏一首乐曲的伴奏或者一条 编写好的节奏, 在演奏的过程中, 加入动作的变换。如: 本来应该在自己鼓 上演奏的拍点, 通过同时左手翻过右手, 右手翻过左手的来回变换, 击打旁
边同学的鼓。形成一种合作式的演奏模式。

3.1.2接龙模式

小朋友任意人数 (不超过 8 人为宜), 演奏一条节奏。可以从第一位也可 以从最后一位开始, 以能达到的最快速度或最慢速度演奏。第一位同学演 奏完成后, 下一位同学接着上一位的速度进行变化演奏 (如上一位是快, 则 渐慢。慢, 则渐快)。

以上环节能充分调动学生积极性, 加强同学之间的合作能力, 合作演 奏的旋律也可由小组组员亲自编创, 寓教于乐, 开发创造力。

3. 2 小木琴

木琴是一种木制的高音特色打击乐器 (木琴有多种规格适应不同年龄 的演奏者, 本文以适合小学课堂的小木琴为例)。小木琴能以琴槌敲击木板 的方式发出具体音高, 是打击乐器中的乐音乐器, 其音色优美灵动, 音域有 三到五个八度的类别可供选择。小木琴的出现, 填补了课堂打击乐器没有 乐音的体系, 这也让多样化打击乐进课堂更具优势和可行性。

在课堂应用中, 木琴可以进行音域跨度不大的乐曲独奏, 也可以胜任 多数乐曲伴奏。作为伴奏乐器, 小木琴具备其他打击乐器所不能比拟的优 势, 它音色新奇, 能让旋律音响更为丰满, 在演奏技法上, 它能够以多槌击 奏和弦。有一定音乐理论的学生能在短时间内演奏出单音和音程, 稍加练 习就能掌握四槌演奏法演奏出七和弦, 呈现多彩的和声音效。

3. 2.1 木琴的教学应用

木琴的教学首先进行的是敲槌练习。

每位同学手握一副琴槌, 手背向上, 用食指第二关节和拇指夹住槌柄, 其他三指轻轻握住槌柄。高举双臂在半空中点槌, 左槌点右槌, 右槌点左槌, 开始时, 可两眼看着击槌, 然后就要求在头顶击槌, 不看槌, 靠感觉去操作。 以此来锻炼对琴槌的掌控能力, 解放双眼去看曲谱和指挥, 凭感觉靠肌肉 记忆寻找位置。

在琴的练习上, 可遵循一定的节奏进行音阶和琶音练习。如全音练习: 以4/4拍, 70的速度, 在一个八度之内进行 11222233 的模进练 习。也可以根据不同要求和需要进行半音模进和其它有规律的、改变拍子 速度和节奏型的模进练习。

\subsection{2 木琴的演奏应用}

在演奏方面, 学生可以在老师的指导或者自主识谱的情况下进行乐 曲演奏。也可以同其它打击乐器如: 非洲鼓、木鱼、三角铁等进行打击 乐合奏。

除了和乐器配合之外, 木琴也能与声乐相结合。可以让几个学生同成 小合唱队, 合唱队同学歌唱时, 木琴进行和声铺垫。

木琴与木琴之间也能进行双声部或多声部的重奏, 选取多声部的卡农 模仿曲谱, 孩子们依次进入。也可选取带伴奏的曲谱, 一部分孩子演奏主旋 律, 另一部分孩子演奏和声。

木琴的加入, 让课堂又增添了一种新的音色和元素, 也让孩子们在旋 律和和声上有了进一步的认识。在往后的学习中, 可以根据自己的音乐理 解, 进行简单创作和和声编配。

\section{3 身体打击乐}

打击乐器之所以种类繁多, 和它本身的属性密不可分。在自然界中, 几乎所有的东西都能用来作为打击乐器。我们随时随地都能从身边拿起一 样物品进行击打, 敲出不同的节奏, 停着即兴的歌曲也能自娱自乐一番。因 此在生活中, 无处不存在打击乐器, 就连我们的身体也能成为打击乐器。身 体打击乐的优势在于随时随地都能演奏, 没有固定的思维, 想到什么就可 以演奏什么。在各大外国教学法中, 身体打击乐的应用极为普遍, 在近年来 
的我国小学音乐课堂中, 也开始逐渐普及起来。

接下来, 介绍一种适用于小学音乐课堂的身体打击乐范例。

首先, 小朋友们按顺序坚排的形式坐在椅子上, 4-5人为一组, 第一位 小朋友面对黑板, 后面的小朋友一次面对前面小朋友的背部, 并闭上眼睛。 由老师或其他没有参与的小朋友在黑板上编写一条由四分音符、八分音 符、十六分音符组合的 $4 / 2$ 拍三小节的节奏。

演奏方式为遇到四分音符和八分音符就要用一只手击打另一只手的 手背。

遇到十六分音符就拍击在大腿上。

节奏编写完毕后, 让第一位同学睁开眼睛, 并给 30 秒的时间, 让其记住 黑板上的节奏, 然后游戏正式开始。

由第一位同学按规定手法演示刚刚在黑板上的节奏, 最多演示两遍。然 后由第二位按照前面的方法依次将第一位同学演示的节奏传递下去。最后 一位同学正确演示出黑板上的节奏为游戏胜利, 反之游戏失败。

根据不同年级, 高年级老师可以让学生可以加入更加复杂的节奏和击 打更多四肢部位的演奏方式。

\section{4 结语}

异彩纷呈、创意无限的特色打击乐器引进小学音乐课堂, 能为小学课 堂现有的感受与欣赏、表现、创造、音乐与相关文化领域教学提供更加丰 富实用的教学活动。打击乐的多样化不仅使课堂形式变得丰富多彩, 也使 学生音乐学习的兴趣得到进一步提升, 在趣中学、乐中学, 更好更快地提升 音乐知识和素养。

就乐器配置上来说, 种类繁多的打击乐器, 既有噪音伴奏乐器, 也有乐 音旋律乐器, 能够较好地进行合作, 依据不同课堂形式、不同学段、不同需 求提供多种教学方式。各类打击乐器大多数也有针对不同年龄层的版本, 价格相对不高。

将多样化的打击乐器引入小学音乐课堂, 无论是使用便捷度、价格、
实用性、适用范围、创作深度上, 都具有明显的优势, 值得在小学音乐课堂 中推广。

\section{[参考文献]}

[1]曹静.谈小型打击乐器教学[J].中小学音乐教育,2014(10):29-31.

[2]敖福森.人体的打击乐器在小学音乐教学中的运用 [J]. 江西教 育, 1988(03):38-39.

[3] 肖娜.打击乐器在教学中的魅力 [J].音乐天地,2004(05):26-27.

[4]朱勇. 让打击乐器敲响学生心中的音符 [J]. 小学教学参 考,2013(24):75.

[5]陈蕴洁. 打击乐器, 奏响合作的旋律一一音乐课堂培养学生合作意 识的实践探索[J].新教育,2016(16):23.

[6]陈净.谈谈打击乐器在低段音乐教学中的作用 [J]. 儿童音 乐,2003(10):39-40.

[7]孙战女.小学低年级音乐课堂中打击乐的有效应用研究[J].音乐时 空,2015(09):182.

[8]黄东升.小学低年级音乐课堂中打击乐的应用探究[J].通俗歌 曲,2017(04):154.

[9]林芳.打击乐在小学低年级音乐课堂中的有效运用[J].福建基础教 育研究,2011(06):110-111.

[10]杨柳.打击乐器对培养低年级学生音乐节奏感的作用分析[J].科 普童话,2017(38):46.

[11]张振华.小学音乐课堂中的节奏训练[J].学周刊,2020(13):149-150.

[12]刘晶.打击乐器在小学音乐教学中的应用[J].学园,2014(21):165. 作者简介:

文沫(1982--), 女, 汉族, 江西南昌人, 硕士研究生, 江西师范大学音 乐学院讲师, 研究方向: 艺术教学论、器乐演奏。 